\title{
Un « smart device » à base de capteur de gaz à nanoparticules
}

\author{
Thomas Nassiet ${ }^{\mathrm{c}}$, Jérémie Grisoliaa ${ }^{\mathrm{a}, \mathrm{c}}$, Jean-Louis Noullet ${ }^{\mathrm{d}}$, Sébastien Lachaize ${ }^{\mathrm{a}, \mathrm{c}}$, Cathy \\ Crouzet $^{\mathrm{a}, \mathrm{c}}$, Reasmey Tan ${ }^{\mathrm{b}}$, François Guérin ${ }^{\mathrm{b}}$, Cherif Rouabhi ${ }^{\mathrm{b}}$, Frédéric Gessinn ${ }^{\mathrm{b}}$, Marc \\ Respaud $^{\mathrm{b}}$
}

${ }^{a}$ Université de Toulouse - LPCNO/INSA, Toulouse, France,

${ }^{\mathrm{b}}$ AIME pôle CNFM de Toulouse, Toulouse, France,

${ }^{c}$ Département de Physique, INSA, Toulouse, France,

${ }^{\mathrm{d}}$ Département de Génie Electrique, INSA, Toulouse, France,

Contact email : jeremie.grisolia@insa-toulouse.fr

\begin{abstract}
Dans le cadre de notre formation pluridisciplinaire à l'INSA TOULOUSE portant sur 1'internet des objets: Innovative Smart Systems, nous avons développé un enseignement complet permettant de réaliser un capteur intelligent à base de nanoparticules. Ce capteur intelligent sert alors de base à la réalisation innovante développée tout au long de la dernière année d'école d'ingénieur et qui est sanctionnée par la présentation devant un jury composé d'industriels du secteur.
\end{abstract}

\section{Introduction}

On assiste à un développement exponentiel des objets connectés (internet des objets) avec un taux de progression estimée de 20 à $30 \%$ sur les 10 années à venir. Cette thématique est au cœur des plans de la nouvelle France industrielle (smart factory, smart grid, smart wearable, smart object, e-health, etc). Aborder ces systèmes nécessite non seulement des compétences techniques pluridisciplinaires mais aussi un sens aigu pour l'innovation et une sensibilité au progrès sociétal. Nous avons mis en place à l'INSA de Toulouse un Parcours Transversal Pluridisciplinaire (PTP), intégrant des groupes d'élèves issus de plusieurs spécialités. Le PTP « Innovative Smart System » (ISS) de l'INSA Toulouse vise la mise en place d'une formation en dernière année d'école d'ingénieur pour former un ingénieur capable d'innover, c'est-à-dire capable de proposer, concevoir, réaliser, diffuser et commercialiser un « smart system » en partant du composant jusqu'à l'application métier en tenant compte des aspects sociétaux.

Un « smart system » intègre des fonctions de détection, d'actionnement et de contrôle afin de décrire et d'analyser une situation et prendre des décisions fondées sur les données disponibles de manière prédictive ou adaptative. Dans la plupart des cas, le « smart system » peut être attribué à un fonctionnement autonome basé sur le contrôle en boucle fermée, l'efficacité énergétique, et les capacités de mise en réseau.

Les nanotechnologies et nanosciences permettent de répondre à ces problématiques car la fabrication de dispositifs à faible coût, haute performance et économes en énergie est l'un de leurs nombreux défis. Un moyen facile de régler les propriétés de ces systèmes est de les construire grâce à des approches bottom-up en utilisant des nanoparticules (NPs) colloïdales synthétisées chimiquement, qui sont accessibles dans différentes tailles, formes, compositions et états de surface. La construction de ces dispositifs permet alors d'exploiter leurs propriétés optiques, plasmoniques, électroniques et magnétiques spécifiques. Les techniques d'assemblage convectif capillaire notamment permettent d'assembler ces nanoparticules à très bas coût, en comparaison avec les coûts engendrés 
par la microélectronique. Par nature, la conduction dans les systèmes granulaires obtenus se fait par effet tunnel, il en résulte des impédances, de l'ordre du M $\Omega$ au G $\Omega$, propices aux systèmes basses consommations. Les capteurs sont alors sensibles aux moindres modifications faites aux jonctions tunnels (distance, environnement électrostatique...), ce qui les rend extrêmement sensibles et permet la réalisation de jauges de contraintes, capteurs de gaz beaucoup plus sensibles que les capteurs usuels.

Pour répondre à ces défis, nous avons développé un module de formation complet intitulé «smart devices » qui se propose de faire réaliser « from scratch » aux étudiants un capteur de gaz intelligent à base de nanoparticules. Dans ce module, les étudiants abordent :

- les principes physiques de fonctionnement et de réalisation pratique de capteurs sur une semaine de stage en salle blanche à l'AIME/CNFM TOULOUSE),

- les procédures de mises en œuvre,

- la conception de conditionneurs,

- la conception du logiciel embarqué dans le microcontrôleur constitutif d'une plateforme open source hardware (Arduino ${ }^{\circledR 1}$ ),

- la conception d'une interface de communication (choix du protocole et logiciel embarqué Android), incluant une base de données.

- $\quad$ et la réalisation de la datasheet du capteur.

\section{Résultats et discussion}

Réalisation du capteur de gaz a nanoparticules:

Le stage technologique pratique de réalisation du capteur se déroule à l'AIME TOULOUSE. Il a pour but de sensibiliser l'étudiant aux exigences et contraintes liées à la synthèse et à la manipulation de nanoparticules préparées par voie chimique (solutions colloïdales), à leur intégration de manière contrôlée et à une application concrète en tant que capteurs de gaz à nanoparticules.

Une première étape consiste à synthétiser des nano-objets : des nano-bâtonnets de WO3. La synthèse se fait en milieu aqueux par une méthode classique de condensation. Ensuite, le procédé de diélectrophorèse développé permet d'intégrer ces nanoparticules au sein de dispositifs électriques (peignes interdigités). Ce procédé est à la fois facile à mettre en œuvre et générique puisque nous l'avons testé avec succès pour différents types de nanoparticules polarisables. Enfin ces dispositifs électriques sont testés en tant que capteurs de gaz. Les mesures sont effectuées sur un banc dédié permettant de faire circuler différents gaz à débit constant dans l'enceinte renfermant le capteur. La température de l'échantillon est contrôlée par l'intensité du courant traversant un élément chauffant, un barreau de polysilicium, intégré à la puce lors de sa fabrication. Les gaz choisis pour la détection sont l'ammoniac et l'éthanol dont la mise en œuvre ne présente aucune difficulté, tous deux à des concentrations de 1000 ppm dans de l'air synthétique.

Conditionnement du signal: un étage d'adaptation d'impédance :

Les caractérisations électriques de ces capteurs montrent que leurs impédances varient de quelques $\mathrm{k} \Omega$ à plusieurs centaines de $\mathrm{M} \Omega$ selon les conditions d'élaboration. Ces fortes impédances imposent l'utilisation d'un étage d'adaptation d'impédance en vue d'une mesure sur une entrée analogique d'un Arduino UNO optimisée pour $\sim 10 \mathrm{k} \Omega{ }^{2}$, nécessitant donc une source de basse impédance.

Notre choix s'est porté sur un étage d'adaptation d'impédance à base d'un amplificateur opérationnel (AOP) très performant et bas coût (quelques $€$ ). La Figure 1 
présente le montage comprenant l'AOP et ses différents filtres, la mesure de courant à travers le capteur de gaz $\left(\mathrm{R}_{\text {sensor }}\right)$, avec une précision de l'ordre du nA. La résistance de calibration $\mathrm{R}_{\mathrm{cal}}=5 \mathrm{k} \Omega$ (changeable grâce aux plots sur la carte PCB (cf Figure 7)) permet de mesurer des impédances allant de $2 \mathrm{M} \Omega$ à $2 \mathrm{G} \Omega$. La résistance mesurée n'est pas la donnée essentielle à la sortie de l'étage d'adaptation d'impédance. En effet, nous préférons étudier une différence relative de résistance entre une valeur témoin préalablement initialisée et la valeur mesurée.

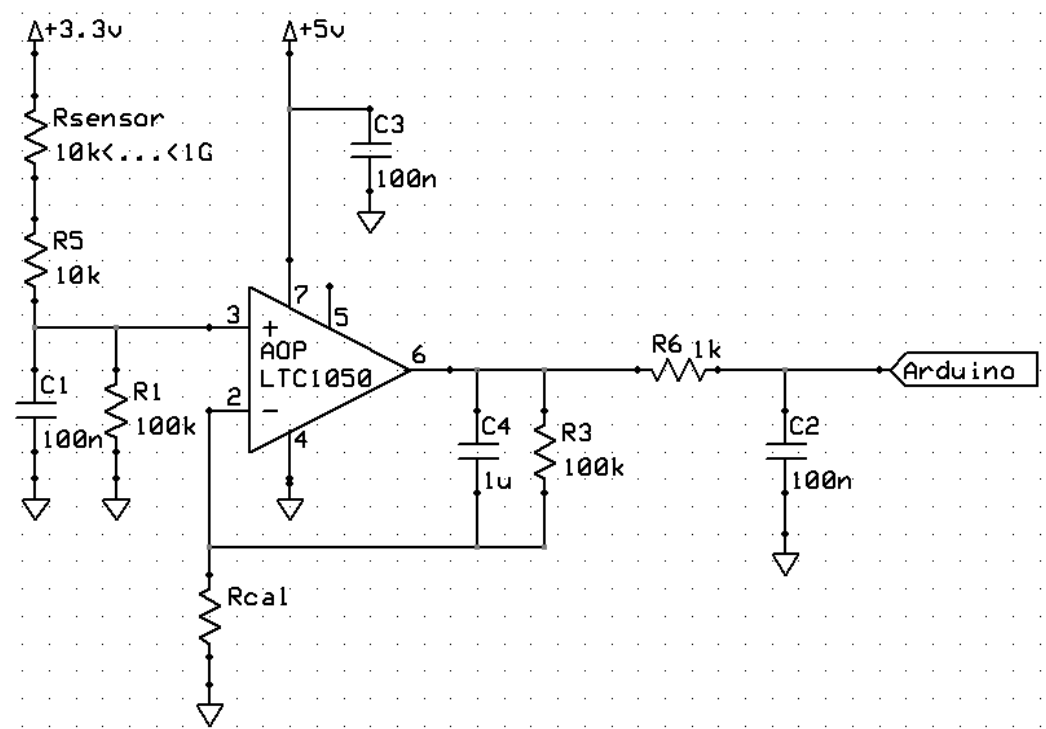

Fig.1 Circuit d'adaptation d'impédance du capteur de gaz

Simulation de validation de l'étage d'adaptation d'impédance :

Le simulateur LTSpice (dérivé de SPICE) est utilisé pour effectuer une simulation servant à présenter et valider l'électronique de traitement insérée entre le capteur de gaz et le microcontrôleur. La Figure 2 représente le schéma d'ensemble.

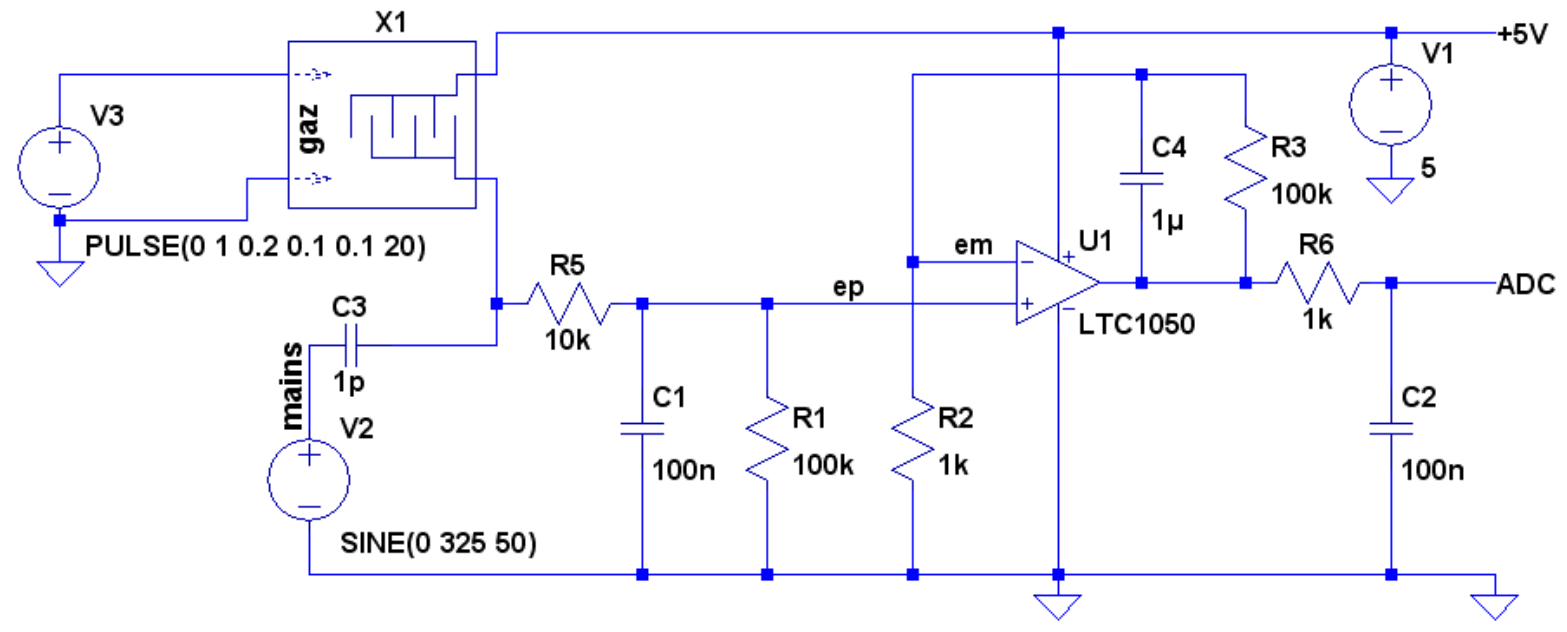

Fig.2 schéma d'ensemble simulé dans LT Spice

Le composant X1 est un modèle du capteur de gaz, qui reçoit de la source V3 un signal représentant la concentration du gaz à mesurer, variant en fonction du temps. La source V2 et le condensateur $\mathrm{C} 3$ modélisent un couplage capacitif parasite avec une ligne d'alimentation $230 \mathrm{~V} / 50 \mathrm{~Hz}$. Ces derniers éléments ont été introduits afin de vérifier que ce 
montage mettant en œuvre de très faibles courants est quand même utilisable sans blindage. Sur la Figure 3, on voit la réponse du système à un échelon de concentration de gaz.

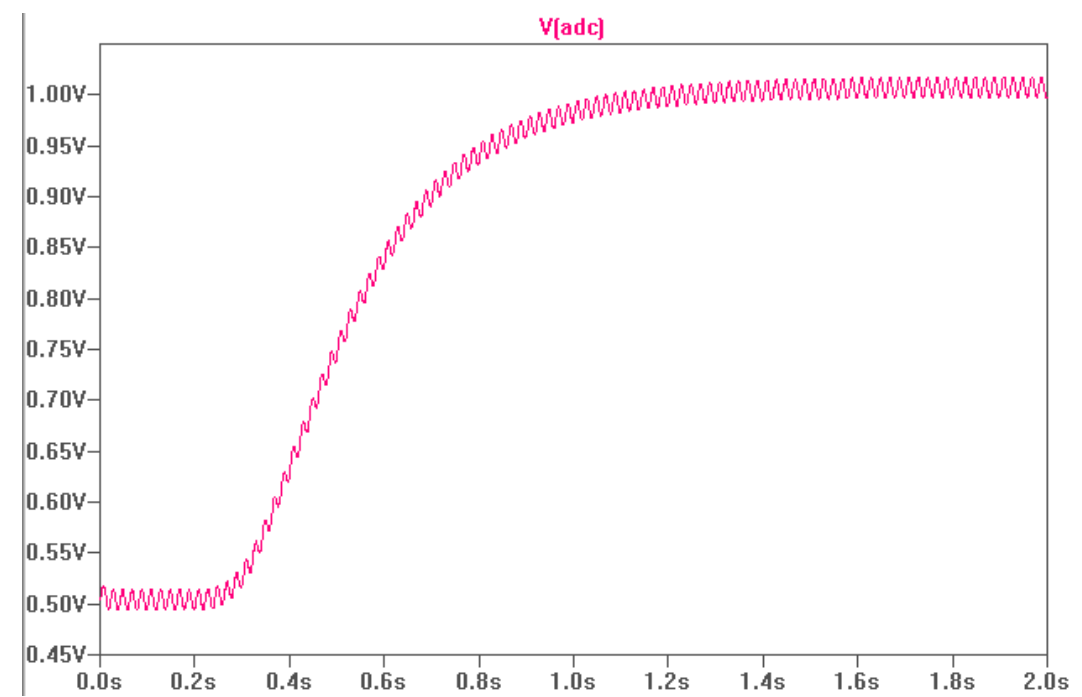

Fig.3 Réponse du système à un échelon de concentration de gaz

L'ondulation parasite à $50 \mathrm{~Hz}$ est atténuée par le filtrage passe-bas inclus dans le circuit $(\mathrm{C} 1, \mathrm{C} 4, \mathrm{C} 2)$, et pourra être complètement éliminée par un filtrage numérique implanté sur le microcontrôleur.

\section{Modèle du capteur de gaz :}

La Figure 4 représente le modèle utilisé pour le composant $X_{1}$. Le cœur de ce modèle est une source de courant B1 définie par une expression algébrique, qui se comporte comme une conductance contrôlée par la tension aux bornes de $\mathrm{C}_{0}$. Le couple $\left(\mathrm{R}_{0}, \mathrm{C}_{0}\right)$ modélise l'inertie du capteur. Les bornes gn et gp servent à introduire une tension représentant la concentration en gaz. Ce modèle est rudimentaire, mais sa structure permettra de le raffiner, par exemple en introduisant dans l'expression du courant une non-linéarité traduisant l'effet tunnel entre les nano-particules.

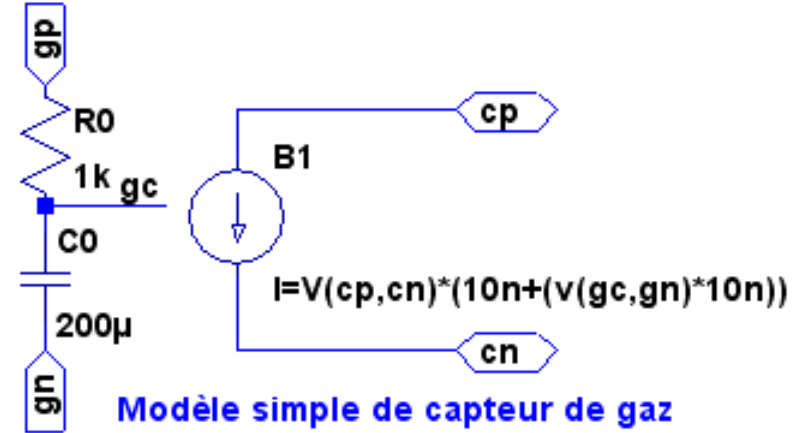

Fig.4 Modèle simple de capteur de gaz

Interfaçage : plateforme open-source hardware Arduino Uno:

La plateforme open-source hardware Arduino ${ }^{\circledR}$ permet de rendre ce capteur intelligent. Grâce à l'utilisation de son microcontrôleur AVR directement programmable par ordinateur et un logiciel associé (IDE), elle permet de récupérer la valeur de la 
tension pour la convertir en résistance (après traitement logiciel éventuel e.g. filtrage, moyennage), l'envoyer à un système sans fil (e.g. Android qui interagira avec une base de données...) et éventuellement effectuer une boucle de rétroaction avec le capteur.

Le montage d'adaptation d'impédance a alors été branché sur l'entrée analogique d'un Arduino UNO permet d'estimer la résistance du capteur (Figure 5).

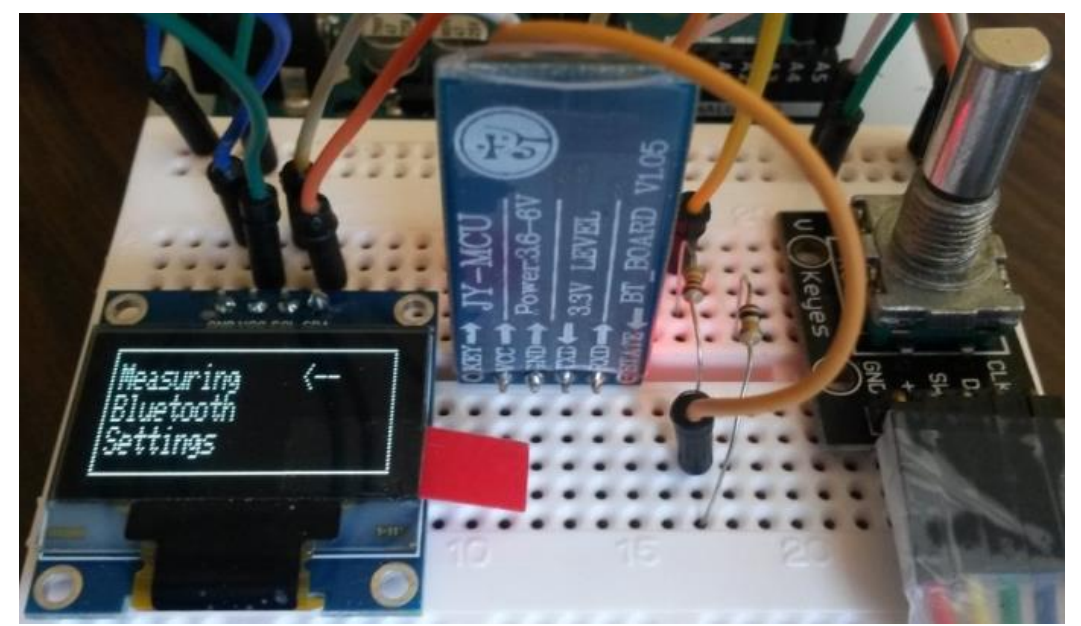

Fig.5 Ecran OLED SSD1306, Encodeur rotatif \& Module Bluetooth JY-MCU

\section{Affichage des données}

Un écran de contrôle, OLED SSD1306 monochrome de 128 par 32 pixels, est connecté à l'Arduino au travers du bus SPI et piloté par une librairie Adafruit. Cet écran permet d'afficher les données du capteur et également possède un système de menus permettant de modifier manuellement la valeur de la résistance de calibration sans avoir à passer par une modification du programme. La navigation dans ces menus est assurée par un encodeur rotatif possédant un bouton de sélection. Par la suite nous avons ajouté un module Bluetooth qui peut aussi être initialisé par ce même programme (Figure 5).

\section{Liaison Bluetooth}

L'ajout d'un module Bluetooth (module JY-MCU) à la carte Arduino rend ce capteur communicant. Ce module utilise deux ports digitaux de l'Arduino comme port série communicant grâce à l'utilisation de la libraire «Software Serial ». Un appariement de ce module Bluetooth à un téléphone Android, lui aussi doté de la technologie Bluetooth est nécessaire avant de pouvoir envoyer les données. Celles-ci sont stockées dans le «buffer » de sortie de l'Arduino et envoyé au smartphone. Un acquittement de l'envoi d'octets du smartphone vers l'Arduino permet d'optimiser la communication entre les deux dispositifs.

\section{$\underline{\text { L'application Android et la base de données }}$}

L'application Android qui reçoit par Bluetooth les données collectées par l'Arduino et les affiche sur un graphique (Figure 6) a ensuite été réalisée grâce à l'application MIT App Inventor ${ }^{3}$ développée par le Massachusetts Institute of Technology (MIT). C'est un outil très simple et très performant permettant de réaliser très rapidement des applications Android en programmant par blocs. Les données collectées par l'appareil Android sont 
ensuite stockées sur la plateforme de stockage de Google qui héberge un script python sur un serveur web.

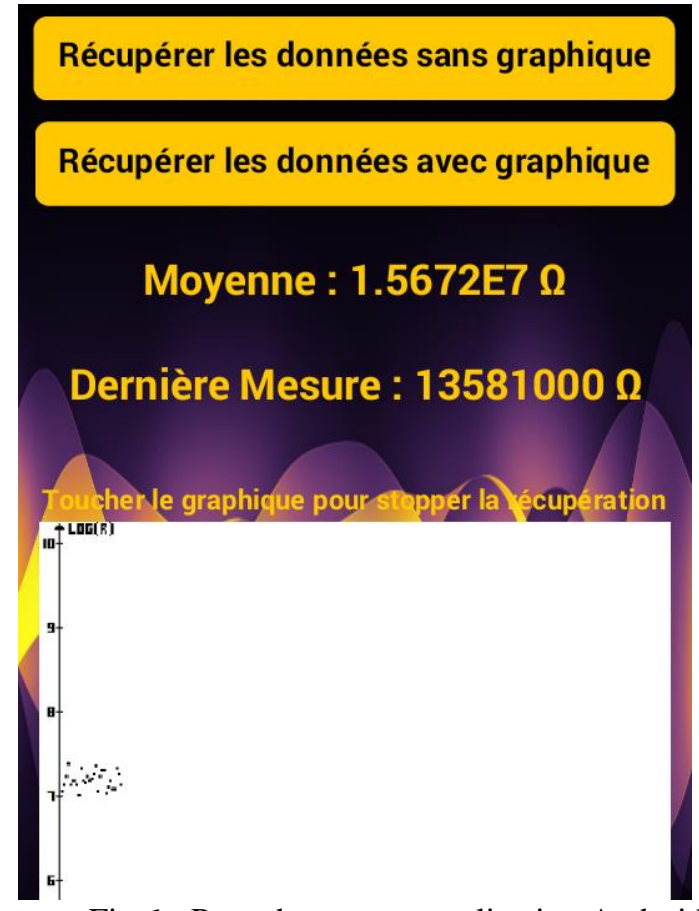

Fig.6 Page de mesure, application Android ISS

«Shield » intégrant les différentes parties

Après quelques phases de tests, les différents modules connectés à l'Arduino et le circuit d'adaptation d'impédance ont ensuite été mis sur une carte électronique PCB (Figure 4).

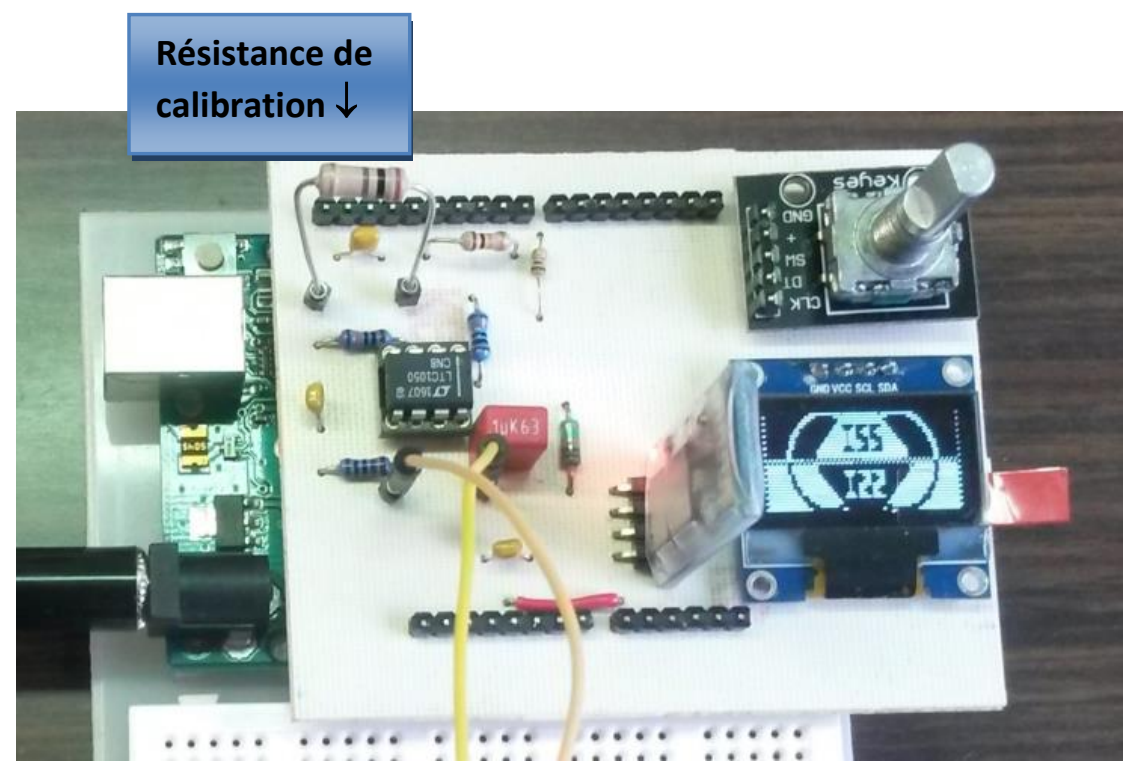

Fig.7 Shield communicant sur plateforme Arduino ${ }^{\circledR}$ 


\section{Conclusion}

Dans le cadre de notre formation à l'INSA TOULOUSE portant sur l'internet des objets PTP ISS, nous avons développé un enseignement complet permettant de réaliser un capteur intelligent à base de nanoparticules. Ce capteur intelligent sert alors de base à la réalisation innovante développée tout au long de la dernière année d'école d'ingénieur et qui est sanctionnée par la présentation devant un jury composé d'industriels du secteur.

\section{Remerciements}

Les auteurs souhaitent remercier Thierry Monteil (DGEI/LAAS) et Daniela Dragomirescu (DGEI/LAAS) enseignants chercheurs à l'INSA de TOULOUSE avec qui nous avons montés le parcours complet PTP Innovative Smart System à l'INSA TOULOUSE, et dont les résultats présentés ici ne représentent qu'un sous-ensemble. Nous souhaitons également remercier TOULOUSE INGENIERIE pour son soutien financier obtenu lors d'un appel à projet. Ils remercient également le GIP-CNFM ainsi

que IDEFI FINMINA ANR 2011 IDFI 0017 pour leur contribution au financement de cette opération.

\section{Références}

1. ARDUINO : https://www.arduino.cc/

2. ATMEGA328 datasheet : http://www.atmel.com/Images/Atmel-42735-8-bit-AVRMicrocontroller-ATmega328-328P datasheet.pdf

3. MIT APP INVENTOR 2: http://ai2.appinventor.mit.edu/ 